\title{
GROUNDED CAPACITOR OSCILLATORS USING A SINGLE OPERATIONAL TRANSCONDUCTANCE AMPLIFIER
}

\author{
MUHAMMAD TAHER ABUELMA'ATTI AND MUHAMMAD \\ HAROON KHAN
}

King Fahd University of Petroleum and Minerals, Box 203, Dhahran 31261, Saudi Arabia

(Received May 27, 1995; in final form July 20, 1995)

\begin{abstract}
New oscillator circuits using operational transconductance amplifiers (OTAs) are presented. Each circuit uses a single OTA and grounded capacitors. The feasibility of obtaining oscillators with independent control of frequency and oscillation is considered. Also, the feasibility of exploiting, to advantage, the frequency dependence of the OTA-transconductance is considered. This may result in OTA-based RC oscillators using only one externally-connected capacitor.
\end{abstract}

\section{INTRODUCTION}

The potentials of the operational transconductance amplifier (OTA) as an integrated circuit building block along with its applications in generating all finite linear circuits were introduced early by Bialko et al. [1]. The OTAs have many attractive features in comparison with operational amplifiers. For example, they require just a few or even no resistors for their internal circuitry, provide highly linear electronic tunability of its transfer gain and have more reliable highfrequency performance.

Over the past few years, a number of schemes have been developed for realizing OTA-based sinusoidal RC-oscillators [2-9]. Most of these circuits use two OTAs in combination with a number of grounded and floating capacitors and resistors. An OTA-based RC-oscillator circuit using a single OTA and grounded capacitors has not been reported in the literature. Such a circuit would be attractive for integration as it uses the minimum number of active elements in addition to grounded capacitors.

It is, therefore, the purpose of this paper to present new OTA-based oscillator circuits using a single OTA, two grounded capacitors and three resistors at most.

\section{PROPOSED CIRCUITS}

Consider the oscillator structure shown in Fig. 1. Assuming an ideal OTA, routine analysis of the circuit yields its characteristic equation given by

$g_{m}\left(Z_{1} Z_{2}-Z_{3} Z_{4}\right)=Z_{1}+Z_{2}+Z_{3}+Z_{4}$ 


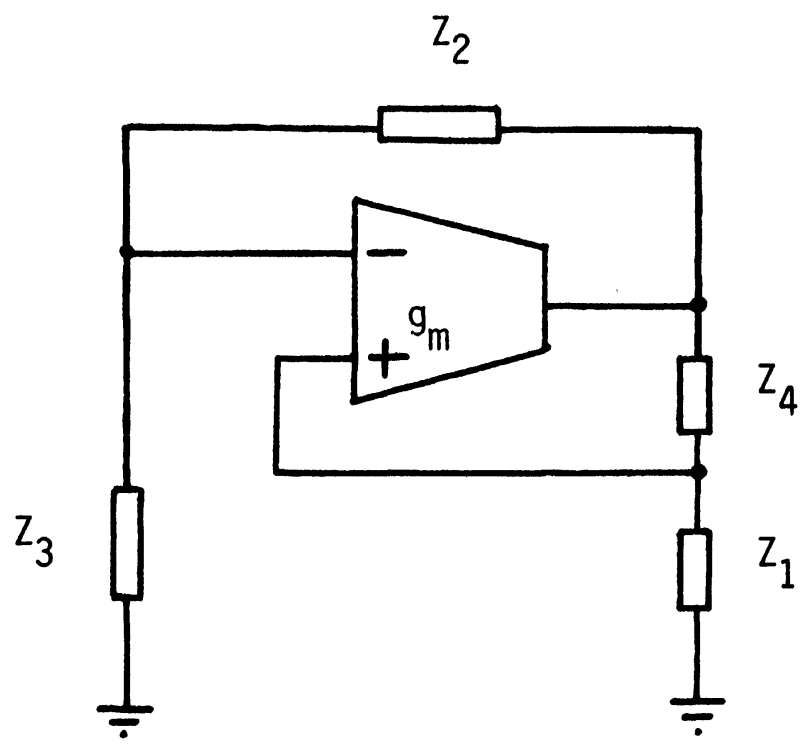

FIGURE 1 Proposed oscillator structure.

Now if we choose $Y_{1}=1 / Z_{1}=G_{1}+s C_{1}, Y_{2}=1 / Z_{2}=G_{2}, Y_{3}=1 / Z_{3}=s C_{3}$ and $Y_{4}=1 / Z_{4}=G_{4}$, shown in Fig. 2(a), equation (1) reduces to

$s^{2}\left(C_{1} C_{3} R_{1}\left(R_{2}+R_{4}\right)\right)+s\left(C_{3}\left(R_{1}+R_{2}+R_{4}\right)+C_{1} R_{1}-g_{m} R_{1}\left(C_{3} R_{2}-C_{1} R_{4}\right)\right)+$ $g_{m} R_{4}+1=0$

By equating the real and imaginary parts of (2) to zero, the frequency and the condition of oscillation of the circuit of Fig. 2(a) can be expressed as
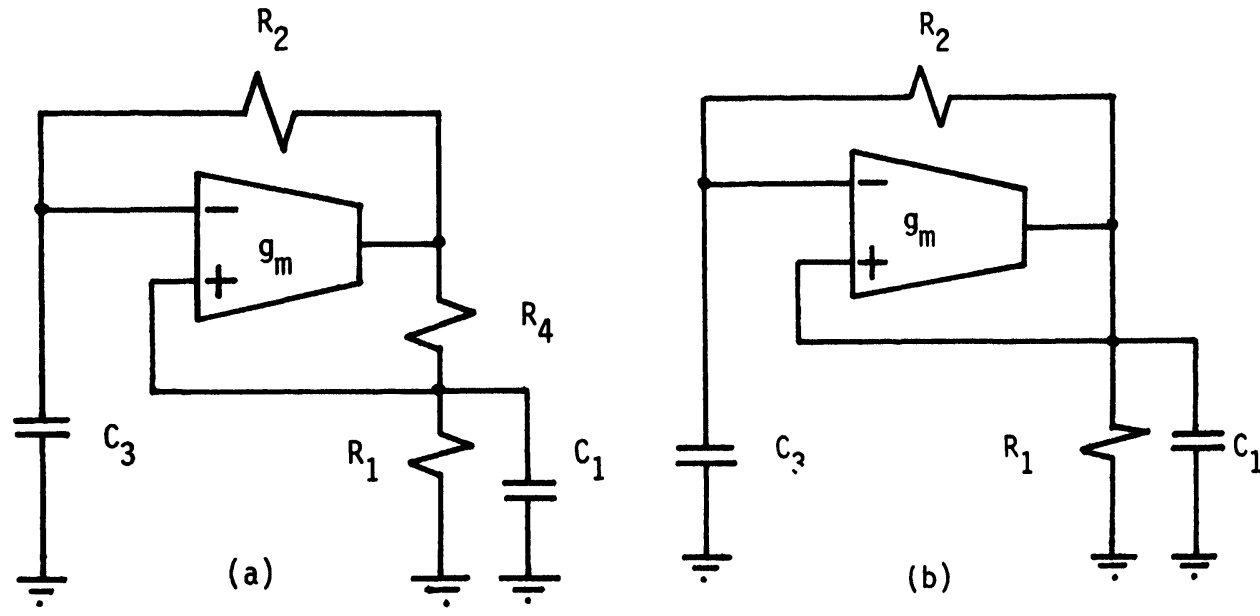

FIGURE 2 Oscillator circuits with grounded capacitors. 
$\omega_{o}^{2}=\frac{1+g_{m} R_{4}}{C_{1} C_{3} R_{1}\left(R_{2}+R_{4}\right)}$

and

$g_{m} R_{1}\left(R_{2} C_{3}-R_{4} C_{1}\right)=C_{1} R_{1}+C_{3}\left(R_{1}+R_{2}+R_{4}\right)$

From (3) and (4) one can see that the frequency of oscillation and the condition of oscillation can be tuned by adjusting $g_{m}$. Thus, the frequency and the condition of oscillation are interdependent. However, if $R_{4}=0$, i.e., short circuit, another oscillator circuit can be obtained. In this new circuit, shown in Fig. 2(b), the frequency of oscillation is given by

$\omega_{o}^{2}=1 / C_{1} C_{3} R_{1} R_{2}$

and the condition of oscillation will be given by

$g_{m} R_{1} R_{2} C_{3}=C_{1} R_{1}+C_{3}\left(R_{1}+R_{2}\right)$

Now if $R_{2}$ is selected such that $R_{2} \ll<R_{1}$, then the condition of oscillation, given by (6), reduces to

$g_{m} R_{2} C_{3}=C_{1}+C_{3}$

From (5) and (7) one can see that the frequency of oscillation of this circuit can be adjusted by tuning $R_{1}$ without disturbing the condition of oscillation, and since $R_{1}$ is a grounded resistor, then obtaining a voltage controlled oscillator is feasible. Also, one can see that the condition of oscillation can be adjusted by tuning $g_{m}$ without disturbing the frequency of oscillation. Thus, the circuit of Fig. 2(b) enjoys independent control of frequency and condition of oscillation if the condition $R_{2}<<$ $R_{1}$ is maintained.

It is worth mentioning here that another oscillator circuit can be obtained from Fig. 1. If we choose $Y_{1}=G_{1}, Y_{2}=G_{2}+s C_{2}, Y_{3}=s C_{3}$ and $Y_{4}=\infty$, shown in Fig. 3 , then (1) reduces to

$s^{2} C_{2} C_{3} R_{1} R_{2}+s\left(C_{3}\left(R_{1}+R_{2}\right)+C_{2} R_{2}-g_{m} C_{3} R_{1} R_{2}\right)+1=0$

and the frequency of oscillation and the condition of oscillation can be expressed as

$\omega_{o}^{2}=1 / C_{2} C_{3} R_{1} R_{2}$

and

$g_{m} R_{1} R_{2} C_{3}=C_{2} R_{2}+C_{3}\left(R_{1}+R_{2}\right)$ 


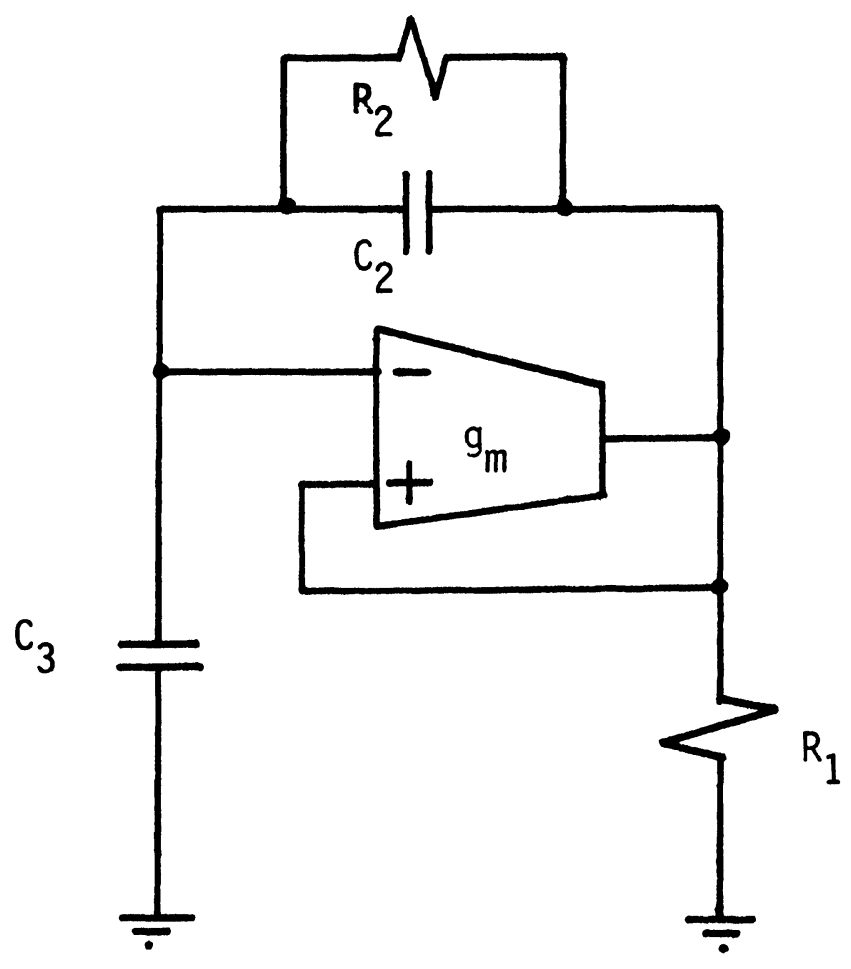

FIGURE 3 Another oscillator circuit obtained from Fig. 1.

From (9) and (10), one can see that the frequency of oscillation and the condition of oscillation are interdependent. However, if we choose $R_{2}>>R_{1}$ equation (10) reduces to

$g_{m} R_{1} C_{3}=C_{2}+C_{3}$

From (9) and (11), one can see that the frequency of oscillation can be adjusted by tuning $R_{2}$ without disturbing the condition of oscillation. Also, one can see that the condition of oscillation can be adjusted by tuning $g_{m}$ without disturbing the frequency of oscillation. Thus, the circuit of Fig. 3 enjoys independent control of the frequency and the condition of oscillation if the condition $R_{2}>>R_{1}$ is maintained. However, the major disadvantage of this circuit is the use of a floating capacitor.

\section{EFFECT OF OTA NONIDEALITIES}

The behavior of the OTA-based oscillators described here may deviate in a number of ways from the ideal operation described by the equations derived in the preceding section. Of particular interest here is the effect of the excess phase shift of the OTA. When the excess phase shift of the OTA is taken into consideration, the transconductance $g_{m}$ of the OTA can be expressed as 
$g_{m}=g_{m o}(1-s \tau)$

where $g_{m o}$ is the transconductance at low frequencies and $1 / \tau$ is the zero of the OTA transconductance-frequency characteristic. Combining (1) and (12), it is easy to show that the conditions of oscillation of the proposed circuits of Figs. 2 and 3 will be

$g_{m o} R_{1}\left(R_{2} C_{3}-R_{4} C_{1}\right)=C_{1} R_{1}+C_{3}\left(R_{1}+R_{2}+R_{4}\right)$

for the circuit of Fig. 2(a),

$g_{m o} R_{2} C_{3}=C_{1}+C_{3}$

for the circuit of Fig. 2(b), and

$g_{m o} R_{1} R_{2} C_{3}=C_{2} R_{2}+C_{3}\left(R_{1}+R_{2}\right)$

for the circuit of Fig. 3.

And the frequencies of oscillation will be

$\omega_{o}^{2}=\frac{1+g_{m o} R_{4}}{C_{1} C_{3} R_{1}\left(R_{2}+R_{4}\right)+g_{m o} \tau R_{1}\left(C_{3} R_{2}-C_{1} R_{4}\right)}$

for the circuit of Fig. 2(a),

$\omega_{o}^{2}=\frac{1}{\left(C_{1}+g_{m o} \tau\right) C_{3} R_{1} R_{2}}$

for the circuit of Fig. 2(b), and

$\omega_{o}^{2}=\frac{1}{\left(C_{2}+g_{m o} \tau\right) C_{3} R_{1} R_{2}}$

for the circuit of Fig. 3.

From (16), one can easily see that if we choose $C_{3} R_{2}=C_{1} R_{4}$, then the frequency of oscillation of the circuit of Fig. 2(a) will not be affected by the excess phase shift of the OTA. Moreover, if the conditions $R_{1}>>R_{2}$ and $R_{2} \gg R_{1}$ are maintained for the circuits of Figs. 2(b), 3 respectively, then the frequency of oscillation and the condition of oscillation of the circuits of both circuits will continue to be independent. Furthermore, it is interesting to note that by exploiting the excess phase shift of the OTA to advantage, it is possible to obtain oscillations using a single externally connected capacitor. For example, from (17) one can see that if $C_{1}$ 
$=0$ then the circuit of Fig. 2(b) can yield oscillation. The frequency of oscillation and the condition of oscillation of the new oscillator circuit, shown in Fig. 4, will be

$$
\omega_{o}^{2}=1 / g_{m o} \tau C_{3} R_{1} R_{2}
$$

and

$$
g_{m o} R_{2}=1
$$

From (19) and (20), one can see that the frequency of oscillation can be adjusted by tuning $C_{3}$ and/or $R_{1}$ without disturbing the condition of oscillation even if the condition $R_{1} \gg R_{2}$ is not maintained. Similar results can be obtained from the circuit of Fig. 3 if $C_{2}=0$. Thus, obtaining a tunable oscillator circuit using a single OTA, two resistors, and one grounded capacitor is feasible.

\section{EXPERIMENTAL RESULTS}

The circuits of Figs. 2 and 3 were tested using the CA3080 OTA. The results obtained are shown in Fig. 5. Good quality sinusoidal oscillations were obtained in

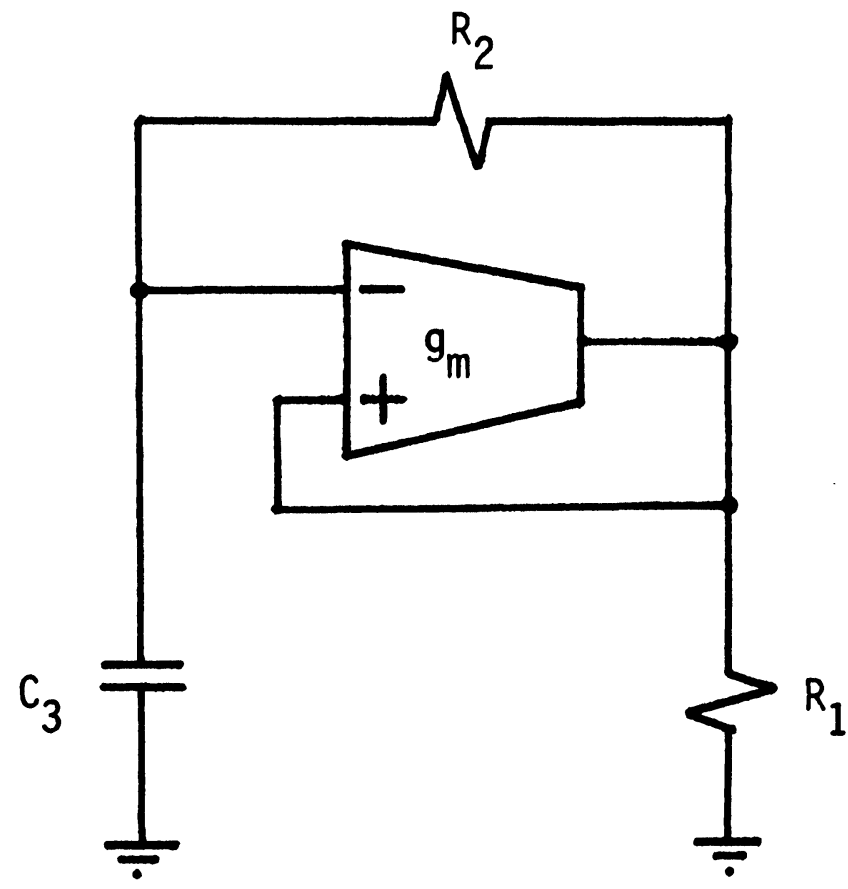

FIGURE 4 New oscillator circuit using only one grounded capacitor. 


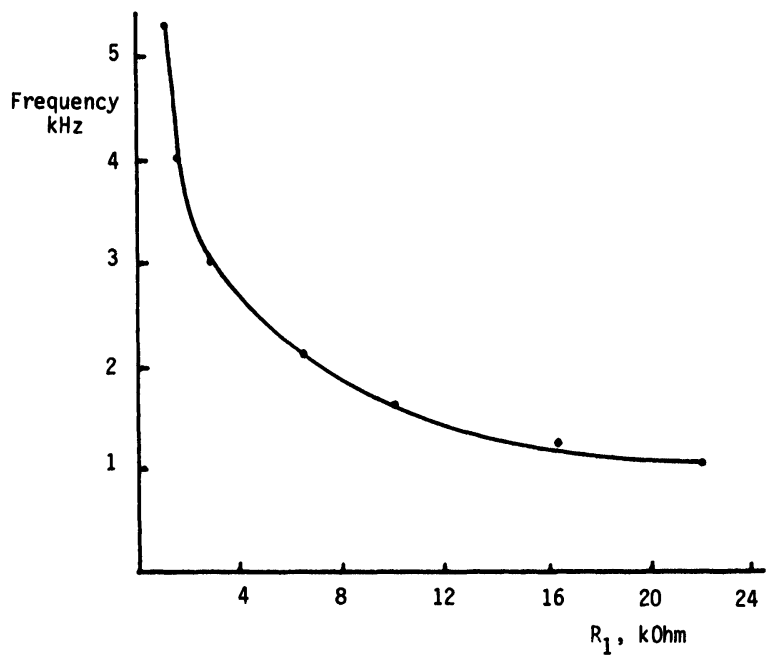

FIGURE 5 Variation of the frequency of oscillation of the circuits of Figs. 2(b) and 3. For Fig. 2(b): $R_{2}=10 \mathrm{k}, C_{1}=C_{3}=10 \mathrm{nF}$. For Fig. 3: $R_{2}=10 \mathrm{k}, C_{2}=C_{3}=10 \mathrm{nF}$.

all cases. The circuit of Fig. 4 was also tested and the results are shown in Fig. 6. It appears that oscillation at relatively higher frequencies can be obtained from this circuit. This is attributed to the dependence of the oscillation on the excess phase shift of the OTA, which will usually manifest itself at relatively higher frequencies.

\section{CONCLUSION}

In this paper, three new OTA-based RC oscillator circuits have been presented. Each circuit uses one OTA only. Some of the circuits use grounded capacitors,

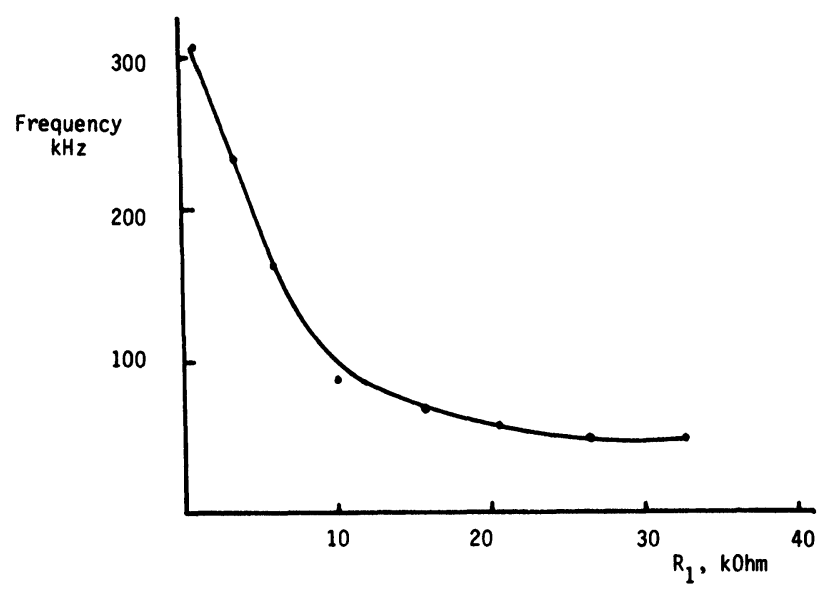

FIGURE 6 Variation of the frequency of oscillation of the circuit of Fig. $4 . C_{3}=220 \mathrm{pF}, R_{2}=10 \mathrm{k}$. 
which is an attractive feature for integration, and also enjoy independent control of frequency and condition of oscillation. By exploiting the frequency dependence of the transconductance of the OTA, it is possible to obtain an OTA-based RCoscillator circuit using a single externally-connected grounded capacitor. This circuit also enjoys independent control of frequency and condition of oscillation.

\section{REFERENCES}

1. M. Bialko and R.W. Newcomb, Generation of all finite linear circuits using the DVCCS, IEEE Transactions on Circuit Theory, Vol. CT-18, 1971, pp. 733-736.

2. M.T. Abuelma'atti and R.H. Almaskatti, Identification of two-amplifiers OTA-based sinusoidal oscillators, Proceedings International Conference on Signals and Systems, Brighton, England, 1989, Vol. 4, pp. 249-260.

3. M.T. Abuelma'atti and R.H. Almaskatti, Novel two-amplifier OTA-based sinusoidal RC-oscillator circuits, Proceedings International Conference on Signals and Systems, Al-Ain, U.A.E., 1990, Vol. 2, pp. 39-48.

4. A.R. Saha, R. Nandi and S. Nandi, Integrable tunable sinusoidal oscillator using DVCCS, Electronics Letters, Vol. 19, 1983, pp. 745-746.

5. J. Hoyle and S.E. Sinencio, Sinusoidal quadrature OTA oscillators, 27th IEEE Midwest Symposium on Circuits and Systems, 1984, pp. 59-62.

6. R. Nandi, Novel variable-frequency oscillator with single resistor control using DVCCS/DVCVS Electronics Letters, Vol. 18, 1982, pp. 144-145.

7. M.T. Ahmed and I.A. Khan, Realization of integrable electronically tunable oscillators with operational transconductance amplifiers, IEEE International Conference on Consumer and Industrial Electronics and Applications, 1984, pp. 20-21.

8. M.T. Abuelma'atti and R.H. Almaskatti, New active-RC sinusoidal oscillators using the operational transconductance amplifier, Proceedings International Conference on Signals and Systems, Miami, Florida, U.S.A., 1989, Vol. 2, pp. 3-8. 

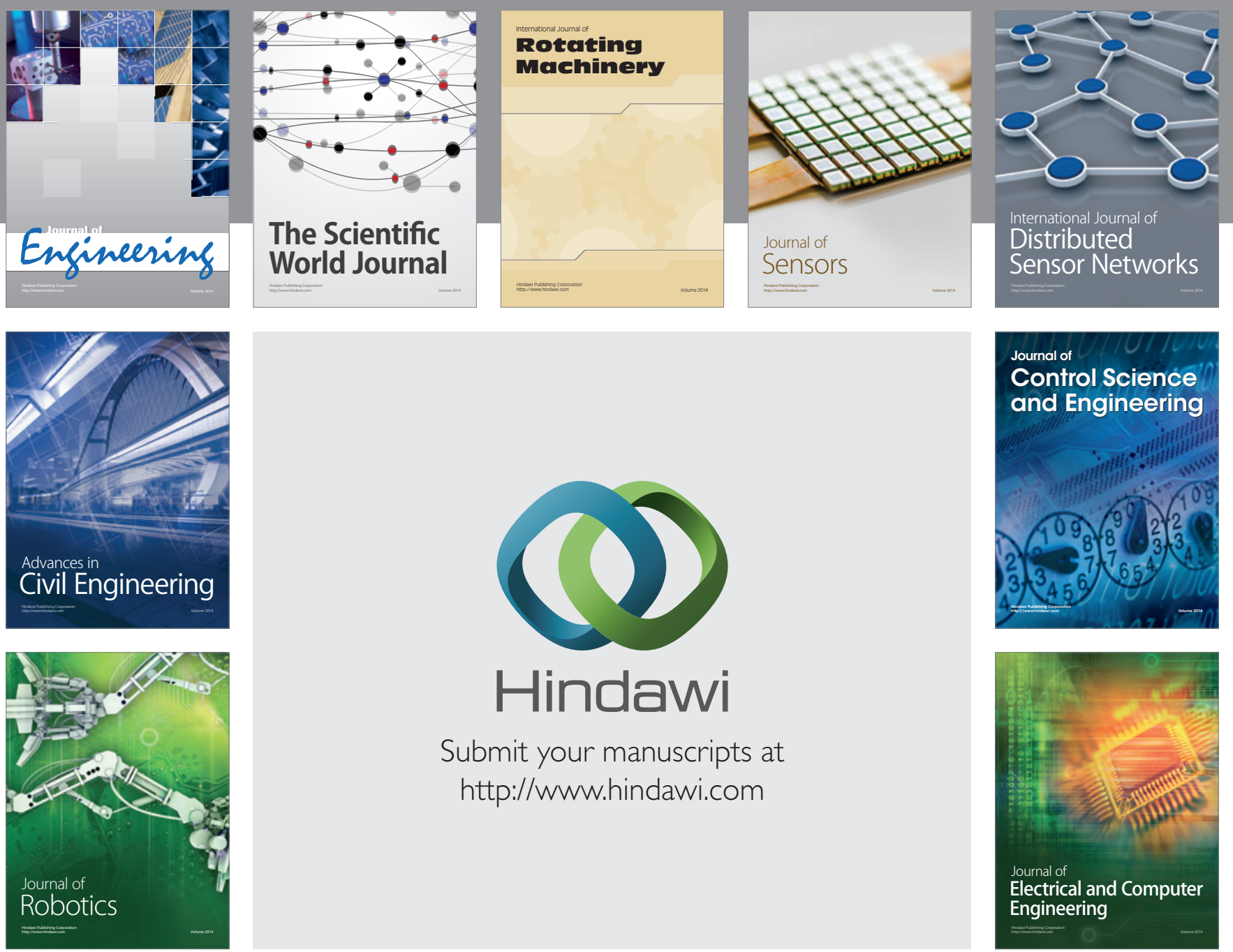

Submit your manuscripts at

http://www.hindawi.com
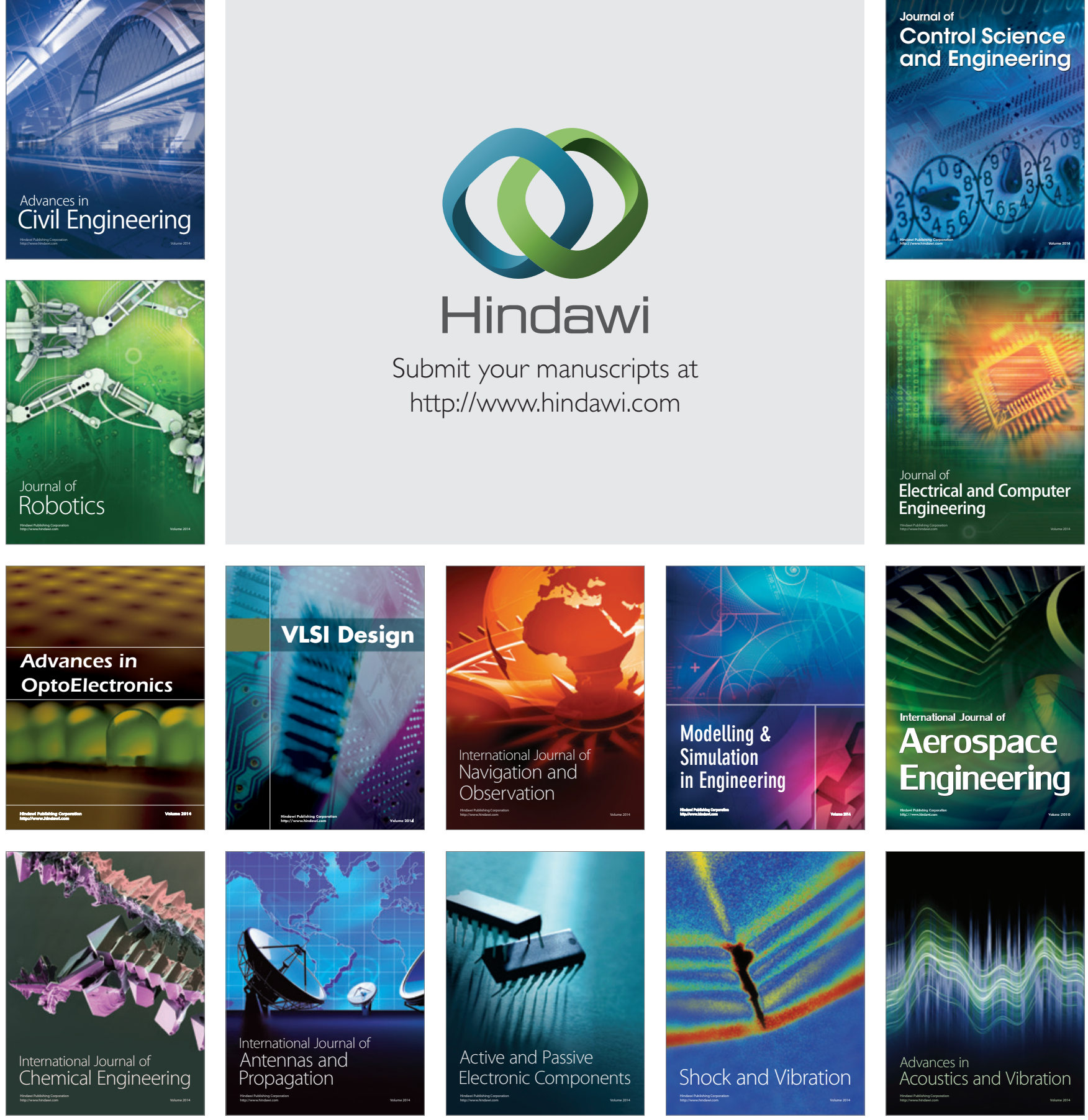